\title{
Research on New Energy and Thermal Power Proportion Comparison of UHVDC Generation Expansion Planning
}

\author{
Licheng $\operatorname{Sun}^{1}$, Gaolei $\mathrm{Wu}^{1}$, Chaoshan $\mathrm{Xin}^{2}$, Lei $\mathrm{Gao}^{3}$, and Zhaowei Geng ${ }^{3}$ \\ ${ }^{1}$ State Grid Xinjiang Electric Power Co., Ltd, Urumqi, 830011, China \\ ${ }^{2}$ Institute of Economic and Technical Research, State Grid Xinjiang Electric Power Co., Ltd, Urumqi, 830002, China \\ ${ }^{3}$ China Electric Power Planning \& Engineering Institute, Beijing, 100120, China
}

\begin{abstract}
Generation expansion planning of UHVDC plays a crucial role in optimizing resource utilization. A mixed delivery of wind, solar, and thermal power can help achieve multiple objectives such as to stabilize power supply, to raise renewable energy ratio, to reduce the wind and solar curtailment rate, while keeping the price acceptable. This paper considers 4 objectives of generation planning of UHVDC and formulates a model to simulate the operation and thus get the key indices that are used to choose from possible generation expansion planning options. We show the effect of the proposed method using a case study of the Xinjiang $3^{\text {rd }}$ UHVDC channel.
\end{abstract}

\section{Introduction}

\subsection{Motivation and aim}

Generation resources and load are reverse-distributed in China. The northwest part of China is abundant in the wind, solar and coal power, and ultra-high voltage direct current (UHVDC) transmission lines are built to transmit the electrical power from northwest to the load centre in the east part. The ratio of power generators for UHV lines starting from the northwest area of China is quite different. For example, the "Hami-Zhengzhou" $\pm 800 \mathrm{kV}$ UHVDC is equipped with $6600 \mathrm{MW}$ thermal power, $8000 \mathrm{MW}$ wind power, and $1250 \mathrm{MW}$ solar power, while the "Zhundong-Wannan" $\pm 1100 \mathrm{kV}$ UHVDC has $13200 \mathrm{MW}$ thermal power, 5200MW wind power, and $2500 \mathrm{MW}$ solar power. To guarantee the economic and effectiveness in future operations, the proportion of different kinds of units must be carefully studied in the planning stage.

Relevant works related to generation planning of UHV lines are reviewed below. Ref [1] illustrates the necessity and feasibility of transmitting large-scale renewable power together with thermal power through UHVDC. An optimization method to determine the capacity of wind and thermal units is provided in $[1,2]$. Ref [3] focuses on the technical and economic analysis of UHVDC units. The proportion of generating units "Jiuquan-Hunan" UHVDC line is studied in [4], where three available options are proposed, and thermal unit equivalent hours and wind curtailment rates are compared. Ref [5] proposes a generation expansion planning model considering environmental policy and ESS facility. However, few papers have examined the multiple objectives together during UHVDC generation planning, and how to compare with available planning options is not provided.

In the practice of generation expansion planning, several available planning options are usually proposed. This paper illustrates how to make comparisons among those possible options considering multiple goals, and find out the best solution.

\subsection{Contributions}

Contributions of this paper are threefold:

1) To examine 4 objectives of the generation expansion planning of a UHVDC, namely power supply ability, renewable energy ratio, new energy curtailment rate and price of electricity.

2) To propose a simplified simulation model to estimate the operation statistics of generating units of UHVDC.

3) To carry out a case study of the Xinjiang 3rd UHVDC channel to find the optimal solution among available generation expansion planning options.

\subsection{Paper organization}

This paper is organized as follows. Section 2 describes the probable objectives during the UHVDC generation expansion planning procedure. An operation simulation model that considers the wind, solar, and thermal power is provided in section 3. A case study of a planned UHVDC starting from Xinjiang province is carried out in section 4. Conclusions are given in section 5 .

\section{Objectives of generation planning of UHVDC}


Multiple objectives should be coordinated in the generation expansion planning of a UHVDC. To be specific, this paper examines the generating units of UHVDC from Xinjiang to Chongqing, also referred to as Xinjiang $3^{\text {rd }}$ channel. In this paper we consider four objectives, including the power supply ability, the renewable energy ratio, the new energy curtailment rate, and the overall price of electricity.

Note that generally these objectives conflict with each other. They may not be optimized simultaneously. The weighted sum method [6] can be used to find the best solution.

\subsection{Power supply ability sub-objective}

To guarantee the power supply of the receiving area, the power flow of the UHVDC is usually kept stable, which does not vary with the wind and solar power among days. Thermal units can help stabilize the stochastic power output of wind and solar power. In this case, we measure the power supply ability according to the capacity of thermal units. The higher capacity of thermal units results in a stronger power supply ability.

\subsection{Renewable energy ratio sub-objective}

China is currently in the rapid procedure of energy transformation. The government has proposed the goal that non-fossil energy will account for $15 \%$ of primary energy consumption by 2020, and $20 \%$ by 2030 . Renewable energy is green and abundant, and thus preferred. In 2019, the renewable portfolio standard (RPS) policy is carried out, which requires electric utilities to gradually increase the amount of renewable energy usage [7]. For UHVDC, the renewable energy ratio should exceed $30 \%$.

\subsection{New energy curtailment rate sub-objective}

The new energy consumption rate is a key index to reflect the usage efficiency of new energy. Due to the low level of local load, lack of power system flexibility in Xinjiang and the imbalance of new energy units and the transmission grid, the wind and solar curtailment rate is high especially in Xinjiang since 2015. Hence, the new energy curtailment rate should be kept within a reasonable level for new generators for UHVDC. Also, a lower curtailment rate usually means more profit for GenCos.

\subsection{Price of electricity sub-objective}

The price of electricity determines whether the UHVDC project is economic. The average electricity price including the transmission price should be no higher than the local benchmark electricity price in Chongqing (the receiving province), and it should meet the agreement signed by both sides.

\section{Model formulation}

To compare with available generation plan options, a simulation model to examine the operation status is formulated below. $t$ is the index for time periods from 1 to $N$, where $N=8760$ for the hours in one year. Superscripts W, S, and I indicate wind, solar and thermal units separately. The capacity of units in each available planning option is regarded as given data.

\subsection{Variables}

Variables include the power output of wind, solar, and thermal units at time $t$, i.e. $P^{\mathrm{W}}(t), P^{\mathrm{S}}(t), P^{\mathrm{I}}(t)$, wind spillage power at time $t, P^{\mathrm{Wc}}(t)$, solar spillage power at time $t, P^{\mathrm{SC}}(t)$, and the power supply from the main grid at time $t, S(t)$.

\subsection{Objective function}

The objective function is to minimize the wind and solar power curtailment and to minimize the power supply from the main grid.

$$
\min \sum_{t=1}^{N}\left(P^{\mathrm{wC}}(t)+P^{\mathrm{SC}}(t)+S(t)\right)
$$

\subsection{Constraints}

Constraints of the proposed formulation is as follows.

$$
\begin{gathered}
P^{\mathrm{I}}(t)+P^{\mathrm{W}}(t)+P^{\mathrm{S}}(t)+S(t)=P^{\mathrm{T}}(t), \quad \forall t \\
P^{\mathrm{min}} \leq P^{\mathrm{I}}(t) \leq P^{\mathrm{max}}, \quad \forall t \\
0 \leq P^{\mathrm{W}}(t) \leq W \times k^{W}(t), \quad \forall t \\
0 \leq P^{\mathrm{S}}(t) \leq S \times k^{S}(t), \quad \forall t \\
-L^{-} \leq P^{\mathrm{I}}(\mathrm{t})-P^{\mathrm{I}}(\mathrm{t}-1) \leq L^{+}, \quad \forall t \\
P^{\mathrm{W}}(t)+P^{\mathrm{WC}}(t)=W \times k^{W}(t), \quad \forall t \\
P^{\mathrm{S}}(t)+P^{\mathrm{SC}}(t)=S \times k^{S}(t), \quad \forall t
\end{gathered}
$$

Eq. (2) is the power balance. Eqs. (3)-(5) limits the power output range for thermal, wind and solar units, where $k^{W}(t)$ and $k^{S}(t)$ are the power output curve factor of wind and solar units, $W$ and $S$ are the capacities of wind and solar for each available plan. Eq. (6) constraints the ramping limits for thermal units. Wind power spillage and solar power spillage are illustrated in Eqs. (7) and (8).

\section{Case study}

In this section, we use a UHVDC in China from Xinjiang to Chongqing to express the proposed method. This UHVDC is still under planning, and is subject to change. The specific figures should be based on actual results. 


\subsection{Data}

The power flow of the UHVDC is shown in Fig. 1, with 5525 equivalent utilization hours per year. Since the sending end of the UHVDC is rich in coal, wind and solar energy resources, we consider a mix of thermal, wind and solar power, and storage may be used if needed. Considering the load requirements and the constraints of the UHVDC operation, we propose three possible options for generation expansion planning. The capacities of each power source are shown in Table 1.

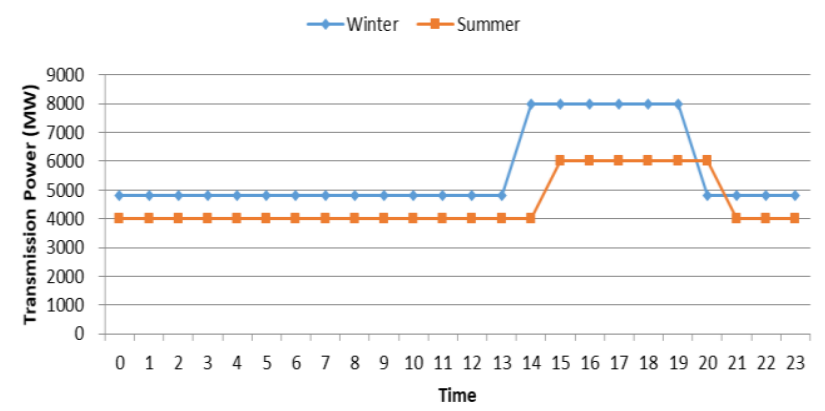

Fig. 1. Transmission power of Xinjiang $3^{\text {rd }}$ channel.

Table 1. Supporting power options of the studied UHVDC (MW).

\begin{tabular}{cccc}
\hline Generation type & Option 1 & Option 2 & Option 3 \\
\hline Thermal power & 6600 & 5940 & 5280 \\
Wind power & 2800 & 4200 & 5500 \\
Solar power & 1400 & 2100 & 2750 \\
Storage & 0 & 0 & 500 \\
Total & 10800 & 12240 & 14030 \\
\hline
\end{tabular}

\subsection{Comparison analysis}

The key indices of the simulation results using the proposed model are shown in Table 2. Option 1 has the highest thermal capacity ratio and thermal energy ratio, the lowest new energy curtailment rate, but the lowest new energy ratio. Option 3 takes the opposite. It has the the highest new energy ratio, but the lowest thermal capacity ratio, thermal energy ratio, and the highest new energy curtailment rate. Indices of option 2 are moderate, which are between option 1 and option 3 .

Table 2. Power supply structure and new energy consumption.

\begin{tabular}{cccc}
\hline & Option 1 & Option 2 & Option 3 \\
\hline $\begin{array}{c}\text { Thermal capacity ratio } \\
\text { (Power supply ability) }\end{array}$ & $61.1 \%$ & $48.5 \%$ & $37.6 \%$ \\
Thermal energy ratio & $78.6 \%$ & $70.0 \%$ & $63.3 \%$ \\
New energy ratio & $21.4 \%$ & $30.0 \%$ & $36.7 \%$ \\
$\begin{array}{c}\text { New energy } \\
\text { curtailment rate }\end{array}$ & $1.2 \%$ & $7.6 \%$ & $13.7 \%$ \\
\hline
\end{tabular}

Since the capacity of thermal units is lower than the maximum transmission power, in some occasions the power supported by Xinjiang's main grid is required, considering the variation of new energy power output. Relevant results of the energy supported are shown in Table 3. In general, energy supply in option 1 is lowest, and that in option 3 is highest.

The results of electricity price analysis are shown in Table 4. In this case, the price of thermal units $(0.256$ yuan $/ \mathrm{kWh}$ ) is the lowest among all the power sources. The higher the thermal power capacity, the lower the total price. The price in option 1 is 0.263 yuan $/ \mathrm{kWh}$. The price in option 2 is 0.265 yuan $/ \mathrm{kWh}$, which is a little bit higher than that in option 1 . The price in option 3 is 0.271 yuan $/ \mathrm{kWh}$, with an added price 0.004 yuan $/ \mathrm{kWh}$ by storage.

Table 3. Energy supported by Xinjiang's main grid (GWh).

\begin{tabular}{ccccccc}
\hline \multirow{2}{*}{ Power from the main grid } & \multicolumn{2}{c}{ Option 1 } & \multicolumn{2}{c}{ Option 2 } & \multicolumn{2}{c}{ Option 3 } \\
& Energy & Percentage & Energy & Percentage & Energy & Percentage \\
\hline $\mathrm{P} \leq 1000 \mathrm{MW}$ & 214 & $47.3 \%$ & 168 & $24.61 \%$ & 118 & $15.3 \%$ \\
$1000 \mathrm{MW} \leq \mathrm{P} \leq 2000 \mathrm{MW}$ & 239 & $52.7 \%$ & 429 & $63.05 \%$ & 322 & $41.8 \%$ \\
$2000 \mathrm{MW} \leq \mathrm{P} \leq 3000 \mathrm{MW}$ & 0 & $0.0 \%$ & 84 & $12.34 \%$ & 330 & $42.9 \%$ \\
\hline
\end{tabular}

Table 4. Electricity price analysis.

\begin{tabular}{ccccccc}
\hline & \multicolumn{2}{c}{ Option 1 } & \multicolumn{2}{c}{ Option 2 } & \multicolumn{2}{c}{ Option 3 } \\
& $\begin{array}{c}\text { Energy } \\
(\mathrm{GWh})\end{array}$ & $\begin{array}{c}\text { Price } \\
(\text { yuan/kWh })\end{array}$ & $\begin{array}{c}\text { Energy } \\
(\mathrm{GWh})\end{array}$ & $\begin{array}{c}\text { Price } \\
(\text { yuan/kWh })\end{array}$ & $\begin{array}{c}\text { Energy } \\
(\mathrm{GWh})\end{array}$ & $\begin{array}{c}\text { Price } \\
(\text { yuan/kWh })\end{array}$ \\
\hline Thermal power & 34.42 & 0.256 & 30.65 & 0.256 & 27.62 & 0.256 \\
Wind power & 7.17 & 0.28 & 9.81 & 0.28 & 12.27 & 0.28 \\
Solar power & 2.16 & 0.31 & 3.06 & 0.31 & 3.59 & 0.31 \\
Main grid power & 0.45 & 0.28 & 0.68 & 0.28 & 0.77 & 0.28 \\
Price without storage & -- & 0.263 & -- & 0.265 & - & 0.267 \\
Storage & -- & 0 & -- & 0 & - & 0.004 \\
Price with storage & -- & 0.263 & -- & 0.265 & -- & 0.271 \\
\hline
\end{tabular}


Table 5. Evaluation of the available options.

\begin{tabular}{ccccccc}
\hline & \multicolumn{2}{c}{ Option 1 } & \multicolumn{2}{c}{ Option 2 } & \multicolumn{2}{c}{ Option 3 } \\
& Value & Score & Value & Score & Value & Score \\
\hline Power supply ability & 660 & 16 & 596 & 15 & 528 & 13 \\
Renewable energy ratio & $21.3 \%$ & 2 & $30.0 \%$ & 15 & $36.5 \%$ & 24 \\
New energy consumption & $2.1 \%$ & 20 & $9.4 \%$ & 16 & $15.3 \%$ & 10 \\
Electricity price & 0.263 & 27 & 0.265 & 25 & 0.271 & 19 \\
Total & -- & 65 & -- & 71 & -- & 67 \\
\hline
\end{tabular}

\subsection{Results}

A comprehensive scoring method considering the four sub-objectives are used to evaluate the available options. The price and renewable energy ratio sub-objectives account for 30 points each, and the NE consumption and power supply ability sub-objectives account for 20 points each. The figures of total points and scoring standard are consistent with the orientation of current national policy in China, and the score for each subobjective is piecewise-linear to its index value. Detailed scoring standards are omitted here due to the length limitation. The results of the scoring are shown in Table 5. Among the three options, option 2 has the highest score and thus recommended in this project.

\section{Conclusion}

The proportion of different kinds of generating units must be carefully studied in the planning stage. This paper proposes 4 objectives of generation planning of UHVDC, which are power supply ability, renewable energy ratio, new energy curtailment and price of electricity. A simple model is proposed to simulate the operation status of the units. Three available options of the generation sources of the Xinjiang $3^{\text {rd }}$ UHVDC channel are compared. Under the given standard, the combination of $5940 \mathrm{MW}$ thermal units, $4200 \mathrm{MW}$ wind units, and 2100MW solar units is recommended.

This work was financially supported by the project "Study on the New Energy Consumption Plan for UHVDC in Xinjiang” (SGXJJY00GHJS190033).

\section{References}

1. Z. Liu, Q. Zhang, C. Dong, L. Zhang, Z. Wang. Efficient and security transmission of wind, photovoltaic and thermal power of large-scale energy resource bases through UHVDC projects. Proceedings of the CSEE. 2014, 34(16): 2513-2522.

2. Z. Wang. Optimization method of uhvdc combined wind-thermal power transmission scale. Electric Power Construction. 2015, 36(10): 60-66.

3. J. Zhang, K. Zheng, H. Huang, et al. The technical and economic analysis on new energy mainly transmitted by UHV DC channels. Electric Power. 2017, 50(06): 152-157.

4. X. Yang, Z. Wang, P. Yang. Study on wind and thermal power bundling of Jiuquan HVDC power sending side. Shaanxi electric power. 2015, 43(06): 83-87.

5. S. Hwang, B. H. Kim, Development of methodology and engineering model for generation expansion planning considering environmental policy and energy storage system. International Journal of Electrical and Electronic Engineering \& Telecommunications. 2020, 9(2): 68-72.

6. Z. Geng, A. J. Conejo, Q. Chen, Q Xia, C. Kang. Electricity production scheduling under uncertainty: Max social welfare vs. min emission vs. max renewable production. Applied Energy. 2017, 193: 540-549.

7. Online: https://www.ndrc.gov.cn/xxgk/zcfb/tz/2019 05/t20190515_962446.html 